Annuaire suisse de politique de développement

23-2 | 2004

Les ONG de développement : rôles et perspectives

\title{
Le rôle des ONG dans la politique de développement : forces et limites, légitimité et contrôle
}

Gérard Perroulaz

\section{(2) OpenEdition}

Journals

Édition électronique

URL : https://journals.openedition.org/aspd/446

DOI : $10.4000 /$ aspd.446

ISSN : 1663-9669

Éditeur

Institut de hautes études internationales et du développement

Édition imprimée

Date de publication : 1 novembre 2004

Pagination : 9-24

ISSN : 1660-5934

\section{Référence électronique}

Gérard Perroulaz, «Le rôle des ONG dans la politique de développement : forces et limites, légitimité et contrôle ", Annuaire suisse de politique de développement [En ligne], 23-2 | 2004, mis en ligne le 08 mars 2010, consulté le 21 septembre 2021. URL : http://journals.openedition.org/aspd/446 ; DOI : https:// doi.org/10.4000/aspd.446 


\title{
Le rôle des ONG dans la politique de développement: forces et limites, légitimité et contrôle
}

\author{
Gérard Perroulaz*
}

\section{Introduction}

L'utilisation du terme «organisation non gouvernementale» (ONG) s'est généralisée depuis plusieurs décennies, alors qu'il ne couvre pas des réalités précises et qu'il est difficile à définir exactement. Les ONG bénéficient d'un grand prestige auprès de la population, mais on connaît finalement assez peu leur travail et le terme même d'ONG est très vague. Se peut-il qu'elles soient si appréciées parce qu'on les connaît finalement si peu ? ${ }^{1}$

Pour ouvrir ce dossier de l'Annuaire, nous nous proposons de relever les difficultés à définir ce terme d'ONG et tenterons d'établir une typologie des ONG de solidarité que l'on trouve en Suisse. Il n'est pas inutile de rappeler certaines forces du travail de ces organisations et certaines limites. Le monde des associations couvre des réalités très diverses; nous nous concentrons ici sur l'examen du rôle des ONG suisses de solidarité internationale.

\section{Essai de définition et de typologie des ONG}

Le terme «ONG» recouvre une très large palette d'organisations de nature différente et il n'existe pas de définition précise et unanimement acceptée de ce que signifie ce terme. Il n'y a pas de définition juridique uniforme dans les différents pays du Nord, et une étude récente montre qu'il serait très difficile de parvenir à une définition commune ne serait-ce que pour les pays de l'Union européenne ${ }^{2}$. Les définitions que l'on trouve peuvent être plus ou moins larges, en comprenant l'ensemble des acteurs non gouvernementaux (y compris les organisations professionnelles, le secteur privé), ou en se limitant, comme nous le faisons dans ce dossier, aux organisations à but non lucratif actives dans la solidarité internationale. L'encadré ci-dessous donne un aperçu des types d'organisations existantes en Suisse. Il faut cependant être conscient que certaines organisations peuvent difficilement n'appartenir qu'à une seule catégorie ${ }^{3}$.

\footnotetext{
Chargé de recherche à l'iuéd. Epd-Entwicklungspolitik, $\mathrm{n}^{\circ}$ 5, 1999.

Voir l'étude sur les difficultés à établir un statut juridique international ou européen des organisations de solidarité internationale, étude menée au niveau de l'OCDE. Commissariat général du plan, L'Etat et les ONG: vers un partenariat efficace, Paris, La Documentation Française, 2002, 210 p. Rapport du groupe «pour un nouveau partenariat entre les organisations de solidarité internationale et les pouvoirs publics », groupe présidé par Jean-Claude Faure.

3 Cet ouvrage contient une bibliographie qui donne des listes d'adresses d'ONG et des portails Internet donnant accès à certaines d'entre elles. Il existe en Suisse plusieurs listes et fichiers d'ONG, dont aucun n'est exhaustif.
} 
Le groupe de travail chargé de conseiller le secrétaire général des Nations unies sur les priorités à fixer pour améliorer l'interaction entre l'ONU et la société civile distingue les acteurs d'Etat ou gouvernementaux, le secteur des affaires privées (entreprises, fédérations d'industries et syndicats patronaux, fondations, médias) et la «société civile». Cette catégorie comprend les organisations de masse représentant les intérêts de groupes particuliers (organisations de femmes, de jeunes, de paysans, de peuples autochtones), les organisations corporatives représentant leurs mandants par la profession qu'ils exercent (syndicats et fédérations internationales les regroupant, associations d'employés de secteur), les organisations religieuses, les universités, les ONG de bienfaisance et les mouvements sociaux (mouvements des paysans sans terre, mouvement altermondialiste, mouvement féministe) $)^{4}$.

La définition des ONG donnée par les Nations unies reste trop large pour être applicable dans tous les pays puisqu'elle inclut des organisations de nature très différente: mouvements sociaux, organisations proches d'Eglises ou des milieux de l'économie privée (associations d'entreprises), centres de recherches universitaires, ainsi qu'associations de parlementaires et d'autorités locales. Plusieurs organisations des Nations unies possèdent des guichets réglant les accréditations des ONG et les relations entre ONG et organisations internationales, sans que les critères soient forcément les mêmes. Le rapport final du groupe de travail recommande notamment de fusionner les procédures d'accréditation en un mécanisme unique placé sous l'autorité de l'Assemblée générale 5 .

Le Conseil de l'Europe a élaboré en 1986 la «Convention européenne sur la reconnaissance de la personnalité juridique des organisations internationales non gouvernementales » (convention 124), mais sa portée est régionale (seuls huit Etats, dont la Suisse, l'ont ratifiée), et elle reste assez vague sur le caractère international et sur l'aspect non lucratif, stipulant simplement qu'une ONG doit «avoir un but non lucratif d'utilité internationale». La convention peut comprendre des organisations de défense d'intérêts professionnels, des syndicats et des organisations comme le Comité international olympique (CIO) ${ }^{6}$.

Pour faire une classification des ONG, une approche différente et intéressante serait de regrouper les ONG autour de certains domaines d'activité, ou pôles de compétences, comme cela a été fait par exemple dans l'étude sur les ONG internationales travaillant autour de la Genève internationale ${ }^{7}$. Cela permet de relever les compétences indéniables d'analyse et d'action dans certains domaines (environnement, droits humains, aide humanitaire, promotion des femmes, commerce international, promotion de la paix). Cet exercice, pratiqué pour les ONG internationales présentes à Genève, reste encore à faire pour l'ensemble des ONG locales présentes en Suisse.

Philippe Ryfman propose de renoncer à des définitions unidimensionnelles, qui ne couvrent souvent qu'une partie de la réalité, pour donner le nom d'ONG aux entités qui réunissent un faisceau de cinq caractéristiques: ${ }^{8}$

4 Source: UNO Panel of Eminent Persons on Civil Society and UN Relationships, <www.un.org/reform/ panel.htm>.

5 Rapport du Groupe de personnalités éminentes sur les relations entre l'Organisation des Nations unies et la société civile, doc. A/58/817, 11 juin 2004, <www.un.org/reform/panel. htm>.

6 Philippe Ryfman, Les ONG, coll. Repères, Paris, La Découverte, 2004.

Voir l'article de Yanik Marguerat dans ce dossier.

Philippe Ryfman, op. cit., pp. 28-30. 
1. La notion d'association (soit le regroupement de personnes privées) avec un projet non lucratif au bénéfice d'autrui.

2. La forme juridique d'association à but non lucratif, selon les droits nationaux.

3. Le fait d'être un espace autonome face à l'Etat ou des puissances privées. L'Etat ne doit pas être à l'origine de la création de l'ONG même si celle-ci peut avoir des liens avec l'Etat. La même autonomie doit exister face à l'économie privée, l'Eglise, des sectes ou des groupes criminels.

4. «La référence à des valeurs impliquant, en même temps qu'un engagement librement consenti, la volonté affichée d'inscrire l'action associative dans une dimension citoyenne insérée dans un cadre démocratique. $\gg^{9}$ L'ONG devient ainsi un des segments de ce que l'on appelle la «société civile».

5. Le caractère transnational de l'action: une action qui est menée dans un autre pays (que le pays d'origine) où elle cherche à défendre les droits humains ou à intervenir pour la protection de l'environnement et le développement durable.

Cette approche permet d'appréhender le monde des ONG avec une certaine prudence, en étant conscient que les organisations utilisant l'étiquette «ONG» peuvent être de nature très différente, telles les ONG pures créations gouvernementales (GONGOs, governmental NGOs; en français ONGOG, organisations non gouvernementales organisées par le gouvernement) ou les organisations mises sur pied par des entreprises ou des milieux défendant des intérêt commerciaux (BINGOs, business NGOs).

Nous nous limitons, dans cet Annuaire de politique de développement, à l'analyse des ONG exerçant une activité de solidarité internationale, surtout des ONG opérationnelles, mais sans oublier celles qui œuvrent dans les domaines de la promotion du développement durable et des droits humains ni les organisations de sensibilisation au développement. Il nous semble en revanche important de distinguer ce que l'on appelle les organisations de solidarité internationale d'autres acteurs non étatiques (Eglises, entreprises, fédérations syndicales), cela sans négliger les rôles importants de ces autres acteurs.

On peut estimer à 10'000 le nombre des institutions d'utilité publique en Suisse $^{10}$. Mille cinq cents institutions de solidarité Nord-Sud sont répertoriées à l'iuéd. Sur la base de ce fichier, qui contient beaucoup d'institutions Nord-Sud qui ne soutiennent pas forcément des projets au Sud, l'iuéd envoie chaque année un questionnaire à plus de 500 organisations non gouvernementales $(\mathrm{ONG})$ pour leur demander une liste des projets conduits grâce aux fonds privés des organisations (sans les contributions des cantons, des communes ou de la Confédération). Les données disponibles et analysées dans cet article sur l'aide privée des ONG portent sur les réponses d'environ 250 organisations qui ont géré des projets à l'étranger. On peut très difficilement avoir un nombre exact d'ONG, car il n'y a pas d'obligation légale en Suisse pour enregistrer une nouvelle association à but non lucratif.

Nous proposons dans l'encadré suivant une classification des ONG d'entraide. Attention: souvent une ONG ne peut être classée dans une seule catégorie. Il

Ibid., p. 29.

10 10’000 ONG est le chiffre avancé par Dieter Pfister, Spendenmarkt Schweiz. 25 Jahre SMS: Die Jubiläumsstudie, Basel, 1994. 
existe beaucoup d'ONG multifonctionnelles, qui possèdent une division spéciale chargée de la coopération au développement, mais qui mènent aussi d'autres activités (aide sociale en Suisse, aide aux réfugiés en Suisse, analyse et débat sur la politique de développement). Il est difficile de montrer l'importance, en nombre d'ONG, de chaque catégorie, mais la grande majorité des ONG suisses - et ce sont aussi les ONG les plus connues du public - appartiennent sûrement aux catégories suivantes: les ONG opérationnelles de coopération ou d'aide humanitaire, les organisations missionnaires (ayant un volet d'entraide), les organisations de défense des droits de l'homme, les associations de solidarité avec un pays donné et les organisations de défense de l'environnement. Face à ces ONG traditionnelles sont apparus de nouveaux acteurs en Suisse au cours des années 1990, dans les domaines de la résolution des conflits et de la promotion de la paix, de la lutte contre la corruption (Transparency International), de la lutte contre le sida, de la microfinance ou du commerce équitable.

\section{Essai de typologie des ONG de solidarité internationales existantes en Suisse}

ـ ONG de coopération au développement et/ou actives dans I'aide humanitaire, organisations menant des activités opérationnelles sur le terrain, en Afrique, en Asie, en Amérique latine ou dans les pays en transition. On peut appliquer une typologie de ces ONG par secteur d'activité (santé, éducation, aide à l'enfance....).

• ONG de récolte de fonds auprès du public. Certaines ONG (comme la Chaîne du bonheur ou Pain pour le prochain) sont actives dans la récolte de fonds, mais ne gèrent pas elles-mêmes des projets et reversent l'argent à d'autres ONG. D'autres ONG représentent des labels de qualité (ZEWO, Bureau central des œuvres de bienfaisance).

- ONG de sensibilisation aux problèmes de développement, lobbies en faveur de changements dans la politique de développement, campagnes relevant le manque de cohérence entre certains instruments de politique extérieure et la politique de développement (ce que l'on appelle en anglais advocacy). Exemples: Communauté de travail des œuvres d'entraide regroupant six organisations (Caritas Suisse, Action de carême, Pain pour le prochain, Swissaid, Helvetas et l'Entraide protestante EPER)a', et la Déclaration de Berne.

- Mouvements antimondialisation ou milieux altermondialistes, autour de thèmes mobilisateurs (antiOMC, opposition aux institutions de Bretton Woods, à l'endettement des pays en développement). Exemples: ATTAC et Action mondiale des peuples (AMP).

- ONG de lobbying et de sensibilisation dans les domaines de l'environnement et du développement durable. Les ONG dans le domaine de l'environnement sont très nombreuses en Suisse, mais seule une partie d'entre elles déploient des activités surtout à l'étranger, tels que le WWF ou Greenpeace. Pro Natura, I'ATE (Association transports et environnement) et Equiterre travaillent surtout sur la Suisse, mais abordent aussi certaines thématiques plus mondiales de développement durable.

ـ ONG développant des capacités d'expertise et d'analyse sur les questions de politique de développement. Bien que la plupart des ONG restent purement opérationnelles et refusent de prendre une quelconque prise de position politique (notamment pour ne pas froisser les susceptibilités de leurs donateurs), certaines ONG ont développé des capacités d'analyse plus larges. Exemples: Pain pour le Prochain, Caritas Suisse ou Swisscontact.

- Groupes de solidarité avec certains pays. Associations composées de représentants des communautés étrangères en Suisse (association des résidants chiliens ou associations de Mexicains par exemple), groupes de solidarité avec certains pays ou certaines régions (Colombie, Tibet, etc.).

- Organisations de commerce équitable: Magasins du Monde, Fondation Max Havelaar, Fondation Step...

- Organisations de défense du droit d'asile et d'aide aux réfugiés. 
- Organisations dans le domaine des droits humains. Certaines ONG internationales sont très connues (Amnesty International, Fédération internationale des droits de l'homme, Human Rights Watch), mais plusieurs autres ONG travaillent notamment autour des travaux de la Commission des droits de I'homme et du Groupe de travail sur les populations autochtones.

ـ Associations de promotion culturelle Nord-Sud.

$\checkmark$ Syndicats.

๑ Eglises et organisations missionnaires.

- Organisations défendant les intérêts de l'économie privée: economiesuisse, par exemple, s'intéresse régulièrement à des dossiers de politique extérieure.

a La contribution de Peter Niggli et d'André Rothenbühler à ce dossier comprend un encadré qui présente les activités de la Communauté de travail.

Sources: Catherine Schümperli Younossian et Gérard Perroulaz (iuéd); Mandat International; CINFO (Bienne).

Les organisations peuvent se regrouper dans des structures formelles de coordination ou participer à des réseaux plus informels ou ponctuels.

\section{Regroupements associatifs, instances de concertation entre ONG}

- Regroupements formels, tels que la Communauté de travail des œuvres d'entraide, Kontaktstelle Umwelt ou la Fondation éducation et développement. Ce sont des collectifs d'associations, des fédérations d'ONG (par exemple fédération sur le plan international de branches nationales), des instances de coordination.

- Regroupements régionaux, soit les fédérations cantonales d'ONG de développement qui existent dans la plupart des cantons romands, la plus grande étant la Fédération genevoise de coopérationa.

- Plates-formes géographiques permettant de regrouper les organisations actives dans un pays particulier ou dans une région (Haîti, Colombie, Brésil, Pérou....).

- Regroupements thématiques, comme par exemple la Coordination Suisse-OMC ou la Maison de l'environnement à Genève.

$\checkmark$ Regroupements informels.

ـ Alliances stratégiques ponctuelles, notamment lors du lancement d'une initiative ou de l'organisation d'un référendum.

- Particulier à Genève: facilitateurs et accueil de délégués non gouvernementaux lors de conférences internationales (par exemple CADONG - Mandat International).

a Voir l'article de Daniel Fino dans ce dossier.

Sources: Catherine Schümperli Younossian et Gérard Perroulaz (iuéd); Mandat International; CINFO (Bienne).

Forces et limites des ONG

Forces des ONG dans la coopération et leur rôle dans la politique extérieure

Les ONG du Nord travaillent avec des ONG partenaires au Sud et sont proches des populations bénéficiaires. Ces ONG seraient donc particulièrement bien placées pour participer aux programmes de lutte contre la pauvreté. La loi sur la coopération de 1976 indique que l'aide doit être destinée en priorité aux pays, populations et régions les plus pauvres, ce qui légitime le soutien financier de la Direction du développement et de la coopération (DDC) à des programmes 
gérés par des œuvres d'entraide. Notons aussi le professionnalisme et les réussites incontestables de nombreux programmes menés au Sud par les ONG. Si certaines ONG ont de la peine à dépasser un niveau très local (ou souhaitent volontairement être efficaces localement), d'autres grandes ONG peuvent toucher des dizaines de milliers de personnes avec leurs programmes dans les domaines de la santé, de l'éducation et du développement rural.

La souplesse et la rapidité d'action qui caractérisent les ONG en font, selon beaucoup, des instruments plus efficaces dans la lutte contre la pauvreté que les gouvernements et les «grands machins», les grandes institutions multilatérales, susceptibles d'être freinés par des lourdeurs bureaucratiques. En outre, les petites ONG montrent une capacité à mobiliser la population pour des actions rapides lorsqu'une crise éclate dans un pays donné. L'exemple de la Roumanie illustre bien comment, au début des années 1990, une multitude d'ONG ont été créées dans un temps record et beaucoup d'initiatives prises en Suisse pour récolter de l'argent et acheminer l'aide à des communautés en Roumanie, alors que seules quelques grandes ONG traditionnelles ont rapidement mis sur pied des actions dans ce pays. Les crises humanitaires entraînent la présence rapide de beaucoup (de trop?) d'acteurs non gouvernementaux qui peuvent être complémentaires aux agences gouvernementales, au CICR et aux organisations internationales. Historiquement, les ONG ont été considérées comme plus efficaces que les agences gouvernementales et les organisations internationales et moins sujettes à soutenir des «éléphants blancs».

Les ONG sont très efficaces pour réunir des dons auprès des populations $d u$ Nord. Les grandes ONG traditionnelles bénéficient d'une base de membres et de donateurs avec en outre, pour les ONG confessionnelles, un ancrage dans les Eglises. De même, les ONG ont la capacité de mobiliser des forces bénévoles au Nord et des volontaires pour le soutien de projets dans les pays du Sud.

Ce travail de proximité et de mobilisation, ce maintien des dons reposent en grande partie sur la confiance accordée par les membres d'une ONG, par les sympathisants et les donateurs. Les ONG bénéficient en général d'un grand prestige auprès du public. Dès les années 1970, elles ont réussi à être considérées comme des porteuses d'espoir, face aux drames humains lors de catastrophes naturelles ou de conflits et face aux inégalités criantes. A l'écart des lourdeurs bureaucratiques des gouvernements et des lois impitoyables du marché qui laisse de côté tous ceux qui n'ont pas de «pouvoir d'achat», elles ont représenté une sorte de «troisième voie».

Les ONG du Nord s'avèrent aussi de précieuses alliées des agences de coopération pour sensibiliser la population à la nécessité de la solidarité Nord-Sud; elles mobilisent une partie de l'opinion publique en faveur de l'aide au développement et servent de canaux d'information sur les projets réalisés dans les pays en développement et sur l'apport de la coopération aux personnes bénéficiaires. Ce rôle de sensibilisation des populations du Nord aux questions de développement et aux problèmes économiques et sociaux dans les pays en développement est très grand.

Advocacy. La possibilité qu'ont les ONG de travailler en réseau sur le plan international et ainsi de se mobiliser en faveur de certaines causes a considérablement renforcé le poids de leurs campagnes, en créant une sorte d' «opinion publique mondiale». Citons les campagnes contre les mines antipersonnel, pour l'accès aux médicaments dans les pays en développement, contre certaines pra- 
tiques d'entreprises transnationales, pour l'allègement de la dette, pour les populations autochtones, pour les droits de l'homme et pour les droits des enfants. Les milieux anti-OMC et les mouvements altermondialistes ont aussi largement exploité les moyens offerts par les nouvelles technologies de la communication, telles qu'Internet et la messagerie électronique. Ce travail d'advocacy, existant déjà pour quelques organisations comme la Déclaration de Berne et la Communauté de travail, s'est élargi grâce à de nouveaux acteurs (ATTAC et l'endettement des pays pauvres, OXFAM et le commerce international...). Dans certains domaines, comme le lobby antiguerre ou les négociations commerciales internationales, les ONG suisses ont joué un rôle important de contrepouvoir; elles ont ainsi dénoncé notamment les exportations d'armes, l'aide liée (crédits mixtes), la promotion de certaines exportations couvertes par la GRE, le non-respect des droits humains ainsi que certaines activités des multinationales, et œuvré en faveur du commerce équitable, du développement durable et de l'éducation au développement.

La politique de concordance en Suisse, la recherche de compromis, le long processus d'élaboration d'une loi (avec les procédures de consultation, les discussions préparlementaires dans les commissions des Chambres fédérales puis les discussions au Parlement), la menace de référendum pour porter certains sujets controversés devant le peuple sont autant de facteurs qui permettent aux ONG de faire valoir leur point de vue sur certaines décisions à prendre. Quelques ONG se sont ainsi nettement engagées dans une participation au processus de prise de décision politique. Les ONG internationales présentes à Genève font un travail semblable en participant aux travaux des conférences internationales.

Les ONG sont devenues, particulièrement dès le début des années 1990, des interlocuteurs sérieux et des partenaires de certains gouvernements du Nord lors des grandes conférences internationales sur le développement social et sur le développement durable. Certaines d'entre elles ont développé une grande aptitude à fournir des expertises, des rapports et des analyses, ce qui a considérablement renforcé leur crédibilité. En Suisse, la reconnaissance du travail des ONG sur le terrain et de leur expertise dans certains domaines a permis aux ONG de devenir un interlocuteur privilégié de la DDC et d'autres offices de l'administration fédérale; les ONG s'expriment lors de procédures de consultation et certaines sont régulièrement consultées par la DDC, le Secrétariat d'Etat à l'économie (seco) et l'Office fédéral des forêts, de l'environnement et du paysage (OFEFP). La qualité du travail d'organisations comme Médecins sans frontières et la Déclaration de Berne dans le domaine de l'accès aux médicaments en fait des interlocuteurs sérieux. On peut évoquer aussi l'expertise d'ONG dans le domaine du commerce équitable, avec par exemple la campagne Clean Clothes, qui permet à ces ONG d'être écoutées à la fois par l'administration fédérale et les entreprises privées.

Les ONG sont devenues un élément important de la gouvernance mondiale, qui n'est plus du seul ressort des gouvernements. D'autres acteurs - société civile, secteur privé, parlementaires - ont un rôle à jouer pour résoudre les problèmes globaux. Les grandes conférences mondiales des années 1990 ont permis de traiter des questions cruciales qui nécessitent une action concertée sur le plan international. Les médias et les ONG ont sans doute largement contribué à sensibiliser l'opinion publique aux enjeux en question. 
La société civile est un acteur important du débat démocratique. Aujourd'hui, la démocratie ne se résume plus, pour les citoyens, à l'élection de parlementaires qui adopteront les lois; on parle de plus en plus de la démocratie participative, qui permet d'intervenir dans le débat politique. Cette intervention inclut les pressions directes des ONG sur les détenteurs du pouvoir et le lobbying auprès de parlementaires lors de discussions au Parlement sur des thèmes qui touchent la politique de développement. Quand les ONG dénoncent publiquement le manque de cohérence des politiques du gouvernement à l'égard des pays en développement, le Conseil fédéral et l'administration publique peuvent être mis sous pression et doivent quelquefois se distancer des positions des ONG ou répondre aux critiques. L'administration publique reconnaît toutefois souvent le rôle important des ONG pour relever l'importance de la coopération au développement et pour mieux prendre en compte les différents aspects de la politique extérieure qui ont des incidences sur les pays en développement (protectionnisme des pays du Nord, développement durable).

Dans ce domaine du lobbying politique, certains succès sont indéniables en Suisse. On peut citer les aspects suivants, non exhaustifs :

$\square$ historiquement, dans les années 1960 et 1970, les débats et les revendications de certaines ONG sur le «maldéveloppement Suisse-monde» ont apporté une contribution non négligeable au processus d'élaboration de la base légale pour la coopération au développement, la loi sur la coopération, adoptée en 1976. Ainsi, certaines organisations non gouvernementales, à l'instar de la Déclaration de Berne, dont le texte fondateur a été rendu public en 1968, et de la Communauté de travail des œuvres d'entraide, créée en 1971, ont eu un rôle précurseur dans la coopération suisse lorsqu'elles ont lancé des campagnes dénonçant des incohérences dans la politique extérieure de la Suisse vis-à-vis des pays en développement et qu'elles sont aussi intervenues de diverses manières en faveur d'une politique extérieure plus ouverte au Sud. Pour beaucoup de mouvements de solidarité avec le «tiers-monde», il s'agissait d'affirmer qu'il ne suffisait pas d'augmenter l'aide en «donnant plus», mais qu'il fallait aussi «prendre moins »;

\ autre succès remporté par les ONG, la pétition sur le désendettement, avec 250'000 signatures récoltées en 1990, a débouché sur l'adoption par le Parlement du crédit-cadre de 700 millions destiné à des mesures de désendettement et de protection de l'environnement. Les ONG ont été étroitement associées à la gestion des fonds de contre-partie, les pays bénéficiant de remises de dettes s'engageant à déployer des ressources financières pour des projets de développement;

$\checkmark$ des campagnes internationales ont permis d'avancer rapidement dans le domaine de la lutte contre les mines antipersonnel.

Mentionnons encore les domaines dans lesquels les ONG de développement mènent des activités de lobbying:

- politique économique, relations économiques et politiques des droits humains : exportations d'armes, relations économiques et de coopération économique de la Suisse avec certains pays (Maroc, Chine, Afrique du Sud et mouvement anti-apartheid), octroi de la Garantie contre les risques à l'exportation pour certains grands projets contestables du point de vue de la politique du développement et de l'environnement (grands barrages); 
$\checkmark$ place financière suisse et fuites de capitaux; endettement international;

$\checkmark$ activités des firmes transnationales par rapport à la politique de développement et à la politique des droits humains. Certaines campagnes de dénonciation de pratiques d'entreprises ont modifié le comportement des entreprises qu'elles visaient, comme la campagne contre les substituts au lait maternel de Nestlé ou celle exigeant le retrait de Triumph de Birmanie;

$\checkmark$ politique d'asile;

$\checkmark$ développement durable;

$\checkmark$ sensibilisation et éducation au développement, valorisation des cultures du Sud;

$\checkmark$ promotion des droits de l'homme;

$\checkmark$ lobbying, au niveau fédéral mais aussi dans certains cantons, en faveur d'une augmentation de l'aide publique au développement et contre les coupures budgétaires dans ce domaine lors des discussions sur les budgets annuels de coopération.

\section{$\square$ Rôle des ONG autour des grandes conférences internationales des Nations unies}

Depuis le début des années 1990, des ONG ont été intégrées dans les délégations officielles de la Suisse (et d'autres pays) auprès de la plupart des conférences internationales: conférence de Rio en 1992, conférence des droits de l'homme à Vienne, conférence du Caire sur la population, Sommet social de Copenhague, conférence Habitat à Istanbul, conférence sur les femmes à Pékin, conférence sur le financement du développement à Monterrey (Mexique).

Le travail des ONG et des Nations unies a permis d'ouvrir de nouveaux espaces de dialogue entre organisations internationales, Etats, syndicats, entreprises et ONG dans des domaines comme la promotion du commerce équitable, la lutte contre le travail des enfants et l'amélioration des conditions de travail. Il ne s'agit plus pour les ONG de se borner à dénoncer certains agissements d'entreprises multinationales par exemple, mais de collaborer avec les autres acteurs pour un meilleur respect de normes environnementales ou sociales ${ }^{11}$.

\section{Limites des ONG}

\section{$\square$ Dissémination des forces due au foisonnement d'organisations}

Small is not always beautiful... Le foisonnement de petites ONG peut être révélateur de la richesse et de la diversité des actions en faveur du développement. Les petites ONG possèdent certes des atouts dans la qualité et la connaissance du terrain et du partenaire au Sud et dans la souplesse et la rapidité de leur action. On connaît par contre aussi les limites de la one-man NGO, organisation ne fonctionnant que sur le charisme et l'enthousiasme du fondateur sans qu'il y ait réellement un travail d'équipe et un partage des prises de décision; cette situation pose de surcroît la question de la durabilité : quel avenir pour l'association quand

11 Dans ce dossier, Yanik Marguerat relève l'importance du travail des ONG internationales autour des conférences et organisations internationales à Genève. 
le leader disparaît? Beaucoup d'ONG sont menées par des directions quasiment familiales ou avec un comité d'association quasiment «invisible», ce qui a l'avantage d'éviter des procédures et des administrations bureaucratiques, mais qui pose le problème de la légitimité de l'organisation et aussi des problèmes de gouvernance. On peut aussi mentionner, pour certaines ONG, le manque de professionnalisme de leur personnel.

Le foisonnement d'ONG renforce-t-il ou non leur «poids politique»? La fragmentation peut empêcher les ONG d'avoir une masse critique suffisante pour faire entendre leur voix. Ce problème du foisonnement se retrouve au niveau des Nations unies: les nombreuses interventions de différentes ONG à certaines conférences internationales (commissions des droits de l'homme par exemple) sont-elles véritablement la manière la plus efficace pour des ONG de faire entendre leur voix, ou faut-il favoriser des coalitions d'ONG? Enfin, le nombre très élevé d'associations démultiplie les tâches administratives.

\section{$\square$ Foisonnements de projets d'ONG dans les mêmes pays et problèmes de coordination}

Le Comité d'aide au développement (CAD) a souvent reproché à la Suisse, dans l'évaluation périodique de l'aide de la Suisse, de ne pas suffisamment concentrer les maigres ressources de la coopération sur un nombre moins élevé de pays. La remarque semble aussi valoir pour les ONG; en effet, non seulement une ONG peut être présente dans plus de pays encore que la DDC, mais un même pays peut voir énormément d'ONG sur son sol. Y a-t-il une trop grande dissémination des forces?

Selon les données disponibles, incomplètes, voici, par ordre décroissant, les pays où l'on note la plus forte présence d'ONG suisses ${ }^{12}$ : Inde (près d'une soixantaine d'ONG suisses), Brésil (une quarantaine d'ONG), Bolivie (au moins 35 ONG), Etats de l'ex-Yougoslavie (une trentaine d'ONG), Colombie et Pérou (une trentaine d'ONG dans chacun de ces pays), République démocratique du Congo, Ouganda, Equateur et Haïti ( 25 organisations environ dans chacun de ces pays).

Pour mieux connaître le travail des uns et des autres, il existe heureusement des plates-formes qui assurent une meilleure coordination entre ONG présentes dans un même pays (Brésil, Colombie, Haïti ou Pérou par exemple).

Ne parlons pas de la situation de certains pays du Sud qui se retrouvent devant la présence d'une vingtaine d'agences gouvernementales de coopération, de dizaines d'organisations internationales, de centaines d'ONG internationales et de milliers d'ONG nationales.

\section{$\square$ Les ONG sont-elles forcément plus efficaces que l'aide gouvernementale?}

Le travail des ONG bénéficie d'un capital de sympathie dans la mesure où il semble à certains moins bureaucratique et plus efficace dans la lutte contre la pauvreté que l'aide bilatérale des gouvernements et l'aide des grandes institutions multilatérales. Nous manquons d'ailleurs aujourd'hui d'analyses et de recherches pour mesurer l'impact réel du travail des ONG dans certains

12 Ne sont comptabilisées ici que les ONG gérant des projets avec des fonds propres, sans les organisations qui gèrent des projets financés uniquement par des collectivités publiques et sans les ONG du pays partenaire qui reçoivent un soutien direct. 
domaines. Des recherches sur la mesure de l'efficacité relative des différents acteurs ${ }^{13}$ restent à faire de manière scientifique et la bonne réputation des ONG est peut-être surfaite. Certains auteurs trouvent aussi que le poids réel des campagnes d'ONG est souvent surestimé et qu'elles n'ont que le pouvoir que les Etats veulent bien leur céder (leurs interventions humanitaires sont par exemple utiles lorsque les Etats ne veulent pas trop «se mouiller» ou en raison de la complémentarité des actions) ${ }^{14}$. Il manque pour l'instant des analyses sérieuses pour évaluer l'impact réel du travail des ONG et leur poids pour influer sur les politiques des droits humains ou les politique d'asile des pays du Nord.

\section{$\square$ Manque de transparence}

La confiance du public dans le travail des ONG doit reposer sur une transparence des activités et des comptes des associations. Les ONG ont souvent critiqué le manque de transparence des activités et des processus de prise de décision des agences de coopération gouvernementales; or, ces mêmes aspects ne sont pas toujours plus clairs au sein de certaines ONG. La transparence financière, la cohérence des actions sur le terrain, la coordination entre acteurs du développement, la reconnaissance des erreurs passées ne devraient pas seulement concerner l'aide bilatérale gouvernementale, mais aussi les acteurs non gouvernementaux. Les bulletins d'information des nombreuses ONG parlent surtout de succès, mais bien peu reconnaissent que l'aide au développement, c'est aussi des échecs, des projets à réorienter, des rapports complexes entre «donateurs» et populations bénéficiaires.

\section{$\square$ Affaiblissement de l'Etat, au Nord comme au Sud}

Les politiques de privatisation dans les pays en développement et de restriction des dépenses de l'Etat dans les domaines sociaux de l'éducation et de la santé se sont accompagnées d'un foisonnement d'initiatives et de créations d'ONG dans bien des pays en développement. Ces ONG n'ont-elles pas pris la place de l'Etat pour certaines tâches où celui-ci devrait jouer un rôle très important?

\section{$\square$ La place des ONG du Sud}

Quelle confiance doit-on accorder aux partenaires du Sud? Les méthodes qu'utilisent parfois les ONG du Nord pour «contrôler» l'utilisation des fonds par les ONG partenaires au Sud se sont alourdies et témoignent d'un manque de confiance envers ces partenaires. Selon certaines ONG, les mécanismes de suivi doivent être légers pour ne pas détourner l'ONG partenaire de son travail essentiel et éviter les lourdeurs bureaucratiques qu'impliquent la rédaction des rapports financiers, des rapports périodiques sur les projets en cours et des rapports finaux sur les projets terminés ainsi que l'accueil de missions d'évaluation. Cependant, le suivi toujours plus précis des projets est de plus en plus souvent demandé par les donateurs du Nord.

13 A ce propos, voir par exemple l'étude de Michael Edwards, David Hulme, Non-Governmental Organisations. Performance and Accountability: Beyond the Magic Bullet, London, Earthscan Publications \& Save the Children, 1998, 260 p.

14 Voir par exemple Samy Cohen, «Les ONG ont déminé la moitié du terrain », Alternatives internationales, $\mathrm{n}^{\circ}$ 6, janvier 2003, ou Sylvie Brunel, «L'humanitaire, nouvel acteur des relations internationales », La Revue internationale et stratégique, n 41 , printemps 2001. 
Le discours sur la coopération évoque le nombre croissant de «partenariats avec des institutions du Sud», le «soutien des initiatives de partenaires » et la nécessité de «répondre aux besoins des partenaires du Sud». Mais, comme le dit un proverbe africain, la main qui donne est au-dessus de la main qui reçoit; c'est tout de même l'ONG du Nord qui a le dernier mot pour choisir de financer tel projet plutôt que tel autre et qui souvent impose ses règles de contrôle ${ }^{15}$. Le mot «partenariat» a-t-il un sens au-delà d'une rhétorique convenue?

\section{$\square$ Comment préserver le capital de sympathie?}

Les ONG bénéficient dans l'ensemble d'un large capital de sympathie auprès du public et l'expertise de certaines ONG est appréciée par une grande partie de l'administration fédérale. Cette confiance risque-t-elle d'être entamée lorsque les médias mettent l'accent sur les manifestations violentes qui ont lieu autour de rencontres internationales telles que les conférences de l'OMC, les sommets du G8, les réunions de chefs d'Etat ou le Forum économique de Davos?

\section{$\square$ Efforts des ONG pour se maintenir en vie}

Les ONG ne sont pas seulement des instruments pour aider les plus pauvres, mais aussi des structures qui ont également des objectifs «d'autoreproduction», pour assurer leur survie et le maintien des emplois. Une des limites de leur travail provient de leur fonctionnement et de la taille importante prise par certaines d'entre elles. Comme toute entreprise et toute organisation, une ONG doit aussi se reproduire, maintenir des activités dans son propre intérêt, pour continuer à financer son secrétariat et son personnel et éviter des restructurations douloureuses. L'ONG n'a pas l' «altruisme» pour seule motivation et elle ne vit pas uniquement dans l'intérêt des pays en développement ou des populations bénéficiaires de l'aide. Pour se reproduire, elle peut adopter les stratégies et le profil qu'elle juge le plus pertinents. Cela explique vraisemblablement le nombre élevé d'ONG travaillant dans les domaines qui attirent le plus facilement les dons privés, domaines de l'aide d'urgence, de l'aide à l'enfance ou de l'aide sanitaire.

\section{$\square$ Craintes face à des prises de position jugées trop politiques}

Comme les ONG dépendent de leurs donateurs et des financements publics, la plupart d'entre elles éviteront d'adopter des prises de position politiques susceptibles de détourner certains de ces donateurs vers d'autres organisations moins «dérangeantes».

\section{Débat sur la légitimité du travail des ONG}

La participation accrue des ONG aux conférences internationales s'est aussi accompagnée d'une médiatisation de plus en plus grande. Ces dernières années sont aussi apparues des critiques émanant de milieux proches de l'économie privée ou proches de l'extrême droite, qui remettent en cause le rôle à leurs yeux excessif donné aux $\mathrm{ONG}^{16}$. Les décisions gouvernementales sont avalisées par les parle-

15 Philippe Demenet, «Les ONG du Sud s'affranchissent du Nord», Le Courrier de l'UNESCO, juilletaoût 2001.

16 Voir notamment le site Internet de NGOWatch, <www.ngowatch.org>, qui est un projet de 1' «American Enterprise Institute for Public Research» et de la «Federalist Society for Law and Public Policy 
ments, qui tiennent leur légitimité des urnes, alors que les ONG seraient créées par des personnes non élues, avec un staff ne devant rendre de comptes à personne (sauf à un comité ou une assemblée générale quelquefois «fantôme»), et peuvent avoir des procédures de prises de décision internes peu transparentes. Manque de représentativité, manque de transparence, trop grande influence de la part de petites minorités, prétention de détenir le monopole de la bonne conscience, corporatisme, ONG du Nord parlant abusivement au nom des pauvres de la planète, voilà des affirmations qui apparaissent régulièrement dans les médias.

Le reproche du manque de légitimité et de représentativité est au cœur du débat public actuel sur les ONG. Certains gouvernements, du Nord comme du Sud, voient d'un mauvais œil que des voix autres que celles des politiciens et parlements s'expriment et cherchent à influencer les décisions internationales. Les régimes dictatoriaux ne supportent tout simplement pas l'existence d'ONG. Face à ce que les uns considèrent comme une richesse du débat démocratique, d'autres gouvernements et politiciens prétendent être les seuls habilités à parler au nom des citoyens, puisqu'ils ont été élus démocratiquement. Selon eux, «ONG» n'est pas synonyme de «société»; les ONG ne défendent pas forcément un intérêt général et représentent un segment seulement de la société, et le terme de «société civile» reste vague («société civile»n'est en outre pas un synonyme d' «ONG»). Enfin, chaque conférence mondiale sur un thème donne lieu à des conférences examinant le suivi des engagements pris (Copenhague +5 par exemple), et les gouvernements du Nord et du Sud n'apprécient pas forcément que la société civile leur reproche de ne pas avoir tenu leurs promesses.

On peut toutefois relever que les parlements et les gouvernements démocratiques n'ont jamais adopté des politiques sans tenir compte non seulement des pressions de leur électorat (il faut bien essayer de se faire réélire), mais aussi de toute une série de lobbies qui cherchent à influencer les prises de décision politiques sur le plan national et international lors des conférences internationales: pensons notamment au lobby puissant de l'économie privée, aux associations professionnelles, aux milieux agricoles, aux milieux religieux, aux syndicats. Il semble donc tout aussi légitime que d'autres segments de la société civile puissent s'exprimer sur des thèmes qui les concernent (mouvements sociaux, organisations de peuples autochtones, ONG d'intérêts public, organisations de bienfaisance, mouvements de retraités, etc.). Les problèmes mondiaux ne peuvent être résolus uniquement par les gouvernements; cette tâche implique l'engagement d'autres acteurs, parlementaires, société civile et entreprises.

En bref, on peut considérer que la légitimité des ONG se construit et se renforce sur les facteurs suivants ${ }^{17}$ :

Studies», proche des milieux conservateurs et de l'économie privée aux Etats-Unis. Les articles publiés sur le site dénoncent par exemple la place trop importante prise par les ONG dans les organisations internationales et leur influence jugée excessive dans certains gouvernements, où certaines ONG assument des rôles quasi gouvernementaux. Selon ces articles, il existe des gouvernements et des parlements élus démocratiquement, qui légifèrent et qui exécutent des décisions; le lobby de la société civile et des ONG jouent un rôle trop important et les pressions des ONG ne sont pas démocratiques. En Europe, des critiques virulentes sur le manque de légitimité des ONG ont été émises par exemple par le ministre des Affaires étrangères français, Hubert Védrine (Le Monde, 18 avril 2001), et dans la presse lors de manifestations anti-OMC ou à l'occasion de rencontres du G8.

17 Voir notamment: Philippe Ryfman, op. cit., pp. 95-104, ainsi que la contribution de Peter Niggli et d'André Rothenbühler à ce dossier. 
- base reconnue, avec un nombre élevé de membres et de donateurs (ne pas être une one-man-NGO et avoir une base de donateur ayant confiance en l'activité de l'organisation);

$\square$ insertion dans une structure fédérative (fédération d'ONG de différents pays) ou être membre d'un réseau mondial important (voir l'impact d'organisations comme Human Rights Watch ou Amnesty International);

- capacité de former des coalitions, de travailler en réseau, d'avoir des relais et des partenaires à l'étranger;

- réussite des programmes menés au Sud, qualité des activités opérationnelles;

$\checkmark$ gestion professionnelle des projets et expertise accumulée dans certains domaines, cette expertise qui fait des ONG, comme on l'a vu, des interlocuteurs reconnus ;

- capacité d'élaborer un projet puis de susciter la mobilisation citoyenne en obtenant un large soutien d'organisations, de groupes sociaux, de partis, de médias, de citoyens (par des pétitions par exemple) pour faire aboutir ce projet.

\section{Contrôle des activités des ONG}

La législation pour créer une nouvelle association en Suisse est particulièrement peu contraignante. Il suffit à deux ou trois personnes de rédiger les statuts d'une association à but non lucratif au sens de l'article 60 du code civil suisse, sans obligation aucune d'inscrire cette association dans un registre du type «registre du commerce».

Quelques scandales sur l'utilisation des fonds récoltés peuvent ternir l'image du travail des ONG. Les cas de malversation sont heureusement rares $^{18}$, mais ils peuvent entacher la réputation de l'ensemble des ONG et rendre les donateurs plus méfiants et donc moins enclins à effectuer des dons. A ce propos, il faut remarquer que dans d'autres pays le contrôle sur les ONG peut être plus grand. En France par exemple, la loi de 1901 impose aux organisations certaines normes minimales en termes de transparence des comptes.

Quelle type de certification faut-il adopter pour les ONG? La question reste controversée parmi les ONG elles-mêmes. Si dans leur quasi-totalité elles ne se montrent pas favorables à un contrôle trop strict de leurs activités par l'Etat, et surtout pas à un contrôle de leur orientation politique, elles sont aussi nombreuses à reconnaitre qu'il est important de ne pas tromper les donateurs et qu'il faut lutter contre les «moutons noirs», les organisations qui précisément trompent le donateur et détournent les dons du financement de projets de développement.

La Suisse possède un système d'autorégulation des ONG : le label de qualité du Bureau central des œuvres de bienfaisance ZEWO, qui concerne 300 organisations environ ${ }^{19}$. Certaines ONG jugent que le système du label ZEWO est adapté

18 La presse a parlé par exemple en 2003 du cas de l'organisation «Ensemble contre les mines » ECM, qui a récolté en Suisse plus de 6,8 millions de francs pour nettoyer des terrains minés, dont 98,7\% ont été utilisés pour des frais administratifs des campagnes de récolte de fonds alors que 100'000 francs seulement ont été versés pour les projets sur le terrain («Spendenmillionen für Afrika versickern in Schweizer Taschen», Tages Anzeiger, 28 juin 2003; «Spenden für die Geldmaschine», Tages Anzeiger, 5 juillet 2003).

19 Le fonctionnement de ce label fait l'objet de l'article d'Ariuscha Davatz dans ce dossier. 
aux organisations d'aide sociale de bienfaisance mais pas du tout aux organisations qui font un travail plus «politique» de sensibilisation aux problèmes Nord-Sud et de lobbying sur les questions de développement et les rapports Nord-Sud. Les organisations de promotion des droits humains et de sensibilisation au développement ne correspondent d'ailleurs pas aux critères d'admission du label ZEWO. Ainsi, ne pas obtenir le label ZEWO ne veut pas forcément dire que l'organisation est peu transparente ou qu'il y a détournements de fonds, mais que les critères d'octroi du label ne correspondent pas au travail particulier de certaines $\mathrm{ONG}$, ou que certaines ONG estiment le coût administratif interne pour répondre aux exigences du label trop élevé, ou encore que la cotisation pour l'affiliation est trop importante. Dernièrement, les quatre Centres sociaux protestants sont même sortis de la ZEWO, jugeant notamment le coût d'adaptation au renforcement de règles de la ZEWO trop élevé20.

D'autres systèmes pour établir des normes de qualité se développent, avec par exemple la certification ISO 9001 pour un management fondé sur la qualité, à laquelle se sont soumises dès 1998 certaines ONG comme Caritas Suisse, Medair, Réalise ou Terre des Hommes Lausanne ${ }^{21}$. La Société Générale de Surveillance (SGS) a pour sa part mis sur pied des critères de contrôle des organisations non gouvernementales, et l'une des premières organisations à obtenir cette certification adaptée de l'ISO 9000 a été Swisscontact en novembre 2003. Sont certifiés les structures internes, le déroulement et les mécanismes de contrôle, et non pas les projets particuliers. Pour Swisscontact, la certification est importante dans un contexte de stagnation des dons, pour améliorer la qualité du travail des $\mathrm{ONG}^{22}$; l'évaluation de Swisscontact par la SGS a été très favorable pour l'ONG qui a obtenu la très bonne quotation «AAA», avec une note de 88 sur 100. Comme le relève l'auteure d'une étude à ce propos, plusieurs facteurs ont poussé les ONG à se soumettre aux exigences d'une certification ISO 9001 :

- l'exigence économique, pour garantir que les ressources allouées à l'ONG soient bien gérées et utilisées conformément aux objectifs fixés par l'ONG;

๖ la recherche d'efficacité;

- la volonté de se démarquer des autres organisations en adoptant un label de qualité et une spécificité;

- l'exigence politique de certains offices fédéraux, avec l'adoption des normes ISO 9001 par le seco;

- la recherche de crédibilité sur un marché saturé, pour montrer aux donateurs et bailleurs de fonds publics que l'ONG met la qualité au centre de ses préoccupations $^{23}$.

20 «Les Centres sociaux protestants renoncent au label ZEWO», Le Courrier, 15 avril 2004.

21 Voir notamment: Justine Rosselet, ONG et management fondé sur la qualité: terre des hommes ou terre des normes?, coll. Itinéraires Etudes du développement, nº 19, Genève, iuéd, 2003, 76 p.

22 «Wie gut arbeiten Entwicklungsorganisationen? Surveillance und Swisscontact präsentieren RatingSystem », Neue Zürcher Zeitung, 13 novembre 2003.

23 Justine Rosselet, op. cit., pp. 43-49. 
Face aux remises en question actuelles du rôle des ONG dans la société civile, il est important que les ONG puissent montrer au public ce que signifie leur travail et l'informer sur les réalisations concrètes sur le terrain. Des milliers de projets de soutien auprès de communautés locales, un soutien à la société civile du Sud, des dizaines de millions de pauvres qui peuvent bénéficier d'une aide dans les domaines de la santé, de l'éducation ou de l'accès à l'eau potable, des actions de reforestation ou de lutte contre la désertification et l'érosion, des centaines de jeunes qui s'engagent dans la solidarité internationale, voilà autant d'initiatives porteuses d'espoir pour le monde qui illustrent le rôle important des ONG dans l'agroforesterie, l'éducation de base, la santé et le renforcement des capacités locales.

Dans les sociétés occidentales, les ONG remplissent aussi un rôle social absolument fondamental en tant que courroie de transmission de valeurs de solidarité, de lutte contre les injustices et les inégalités. L'action des ONG est sans doute une composante essentielle de ce que l'on appelle la société civile et un élément important des mouvements sociaux, aux côtés des mouvements syndicaux, des mouvements féministes et de ceux qui luttent contre les discriminations et contre la pauvreté. La vivacité et le dynamisme du monde des ONG permettent aussi de rêver qu'un «autre monde est possible». Les actions menées au Nord pour informer le public sur le problème persistant de la pauvreté, pour dénoncer le manque de cohérence des politiques des pays du Nord à l'égard du Sud, pour montrer aussi le soutien que l'on peut apporter aux populations pauvres par des projets concrets constituent autant de domaines où les ONG ont un rôle important à jouer, complémentaire au rôle d'information des médias, complémentaire aussi à l'action des gouvernements et des grandes agences multilatérales.

\section{SOURCES}

\section{Ouvrages et études}

Comeliau Christian, L'émergence internationale des organisations non gouvernementales, coll. Itinéraires notes et travaux, $\mathrm{n}^{\circ} 68$, Genève, iuéd, 2003, 25 p.

Deler J.-P., Fauré Y.-A., Piveteau A., Roca P.-J., ONG et développement. Société, économie, politique, coll. Hommes et Sociétés, Paris, Karthala, 1998, 684 p.

Freymond Jean-F., Boyer Brook, Les organisations non gouvernementales et la politique extérieure de la Suisse. Rapport de synthèse, Genève, CASIN (Centre d'études pratiques de la négociation internationale), 1998, $22 \mathrm{p}$.

Organisation des Nations unies, Rapport du Groupe de personnalités éminentes sur les relations entre l'Organisation des Nations unies et la société civile, 58 ession de 1'Assemblée générale de l'ONU, Renforcement du système des Nations unies, doc. A/58/817, <www.un.org/reform/panel.htm>.

Ryfman Philippe, Les ONG, coll. Repères, Paris, La Découverte, 2004, 124 p.

Article

Niggli Peter, Rothenbühler André, «ONG et gouvernance mondiale. Une légitimité contestée», article paraissant dans le présent dossier. 